\title{
Predicting initial margin status in breast cancer patients during breast-conserving surgery
}

This article was published in the following Dove Press journal: OncoTargets and Therapy

\author{
Zihao Pan ${ }^{1,2, *}$ \\ Liling Zhu',2,* \\ Qian $\mathrm{Li}^{1,2, *}$ \\ Jianguo Lai ${ }^{1,2}$ \\ Jingwen Peng ${ }^{3}$ \\ Fengxi Su ${ }^{1,2}$ \\ Shunrong $\mathrm{Li}^{1,2}$ \\ Kai Chen ${ }^{1,2}$ \\ 'Guangdong Provincial Key Laboratory \\ of Malignant Tumor Epigenetics \\ and Gene Regulation, Sun Yat-sen \\ Memorial Hospital, Sun Yat-sen \\ University, ${ }^{2}$ Breast Tumor Center, \\ Sun Yat-sen Memorial Hospital, \\ Sun Yat-sen University, ${ }^{3}$ Department \\ of Rehabilitation Medicine, Sun Yat- \\ sen Memorial Hospital, Sun Yat-sen \\ University, Guangzhou, China \\ *These authors contributed equally \\ to this work
}

Correspondence: Kai Chen; Shunrong Li Breast Tumor Center, Sun Yat-sen Memorial Hospital, Sun Yat-sen University, 107 Yan Jiang West Road, Guangzhou, Guangdong 510120, China Email chenkai23@mail.sysu.edu.cn; lishr@mail.sysu.edu.cn
Background: We sought to develop and validate a model for prediction of initial margin status during breast-conserving surgery (BCS).

Methods: We included eligible breast cancer patients receiving BCS in Sun Yat-sen Memorial Hospital from January 2003 to December 2014. All patients received intraoperative frozensection analysis for initial margin assessment. We used univariate and multivariate logistic regression analyses to screen for predictors. A nomogram was developed in the training cohort $(n=1,193)$ from the south branch of the hospital and externally validated in the validation cohort $(n=499)$ from the north branch. We used the area under the receiver-operating characteristic curve and Hosmer-Lemeshow tests to assess the discrimination and accuracy of the nomogram.

Results: The initial margin-positivity rates were $19.5 \%$ and $25.2 \%$ in the training and validation cohorts, respectively. Preoperative tumor size, preoperative lymph-node status, suspicion of multifocality, hormone-receptor status, and HER2 status were significantly associated with margin status. The model included these five variables. The discrimination and calibration of the model were considered acceptable in both cohorts.

Conclusion: The nomogram can predict the likelihood of having positive initial margins during BCS and may be useful for clinical decision-making in the surgical treatment of breast cancer patients.

Keywords: breast cancer, breast-conserving surgery, margins, nomogram

\section{Introduction}

Breast-conserving surgery (BCS) is a standard surgical treatment for early-stage breast cancer patients. The National Surgical Adjuvant Breast and Bowel Project B06 and the Milan study demonstrated that patients undergoing BCS with radiotherapy exhibited survival rates equivalent to those undergoing mastectomy. ${ }^{1-3}$ A major concern for women receiving BCS is the risk of local recurrence. Risk factors of local recurrence after BCS include younger age, HER2-positive disease, and inadequate surgical margins. ${ }^{4-11}$ Among these risk factors, the margin status is most important and the only factor that surgeons can control.

To obtain negative margins, wide local excision with a rim of macroscopically normal tissue around the tumor is necessary. However, $20 \%-40 \%$ of patients have positive margins and require a second surgery for reexcision. ${ }^{12}$ Therefore, numerous approaches have been suggested to improve BCS and reduce the margin-positivity rate. ${ }^{13}$ Frozen-section analysis (FSA) is one of the most useful methods. ${ }^{14,15}$ We have reported that the rate of second surgery for reexcision was only $3.5 \%$ after BCS when FSA was used. ${ }^{16}$

Despite the low second-surgery rate, $15 \%-25 \%$ of these patients exhibit positive initial margins based on intraoperative FSA during BCS. Intraoperative reexcision or 
mastectomy is required in these patients to achieve negative surgical margins. If the surgeon can predict the likelihood of a patient having a positive initial margin, a different surgical approach can be determined preoperatively to reduce operation time and medical costs. ${ }^{17,18}$ In this study, we aimed to develop a nomogram to predict the risk of positive initial margins during BCS. This nomogram will be informative for clinical decision-making.

\section{Methods}

\section{Patients}

We searched the database and deidentified eligible breast cancer patients from the south and north branches of Sun Yat-sen Memorial Hospital. Inclusion criteria were having undergone BCS between January 2003 and December 2014, patients that converted to mastectomy due to positive margins after the first BCS attempt had been included, received an FSA assessment of six to eight margins around the residual cavity intraoperatively, critical information was available, and patients with pure ductal carcinoma in situ (DCIS) and invasive cancer. Exclusion criteria were metastatic, de novo, and stage IV patients and phyllodes tumors of the breast.

For eligible patients, clinicopathological features collected were preoperative tumor size (ultrasound/physical examinations), tumor laterality, preoperative nodal status, suspicion of multifocality (assessed on radiography), initial margin status (collected from the pathology report of the intraoperative FSA), presence of DCIS on needle biopsy, histological type, age, hormone receptor (HR) status, HER2 status, and neoadjuvant chemotherapy (NAC). Preoperative nodal status was assessed using clinical, radiological, and pathological examinations to determine whether a biopsy was required. Suspicion of multifocality was defined as the presence of two or more tumor foci within the same quadrant of the ipsilateral breast assessed using radiography. This study was reported based on the STARD guidelines. ${ }^{19}$

\section{Operation and pathology}

BCS and margin assessment were conducted as previously described. ${ }^{20}$ Briefly, we removed a $1 \mathrm{~cm}$ rim of macroscopically normal tissue around the tumor during BCS to ensure the grossly negative margin. The resection was expanded downward to the pectoralis major fascia and up to the subdermal plane of the skin. After removing the tumor-containing specimen, six to eight cavity margins were systematically collected around the residual cavity and submitted for pathological evaluation. Each of the submitted initial margins was transferred into one block, and two slides were examined in the intraoperative FSA. A margin with components of invasive carcinoma or DCIS intraoperatively was defined as an initial positive margin. Additional excision was performed when one or more initial positive margins was reported. Wider excisions were made on the corresponding sites where the cavity margins were positive until negative margins were ultimately obtained. The decision to perform intraoperative reexcision and collect a new margin for FSA or to convert to mastectomy was determined by the surgeons based on their personal judgment and patients' preferences (collected before surgery). All margins underwent formalinfixed and paraffin-embedded pathological examinations postoperatively. HR positivity was defined as ER positivity or PR positivity, defined as any proportion above zero of positive tumor cells with nuclear staining. We used HercepTest to determine HER2 status. ${ }^{21}$ HER2 was positive if a score $\geq 3$ was obtained by immunohistochemistry or a score $\geq 2$ with HER2 amplification was obtained via fluorescence in situ hybridization. HercepTest scores of 0 and 1 (or 2 without HER2 amplification via fluorescence in situ hybridization) were considered HER2-negative.

\section{Statistical analysis}

A descriptive analysis of baseline clinicopathological features was conducted. Continuous variables were reported using medians and ranges, and categorical variables were reported as percentages. Since only a few patients with impalpable masses had been treated at our institution, we categorized these patients into the preoperative size $\leq 2 \mathrm{~cm}$ category. If the patient had undergone excision biopsy, preoperative tumor size was reported as unknown. We used univariate logistic regression analysis to screen for predictors of positive margins. Significant predictors were included in the multivariate logistic regression analysis. Significant predictors from the multivariate analysis were included in nomogram development. We used receiver-operating characteristic curves and calibration plots with the HosmerLemeshow goodness-of-fit test to assess the discriminative power and accuracy of the model, respectively. Patients from the south and north branches were considered the training and validation cohorts, respectively. $P<0.05$ was considered significant. Data analyses were performed with Stata version 13.1 (StataCorp LP, College Station, TX, USA), and R version 2.11.1 (R Project for Statistical Computing, Vienna, Austria) was used to build the nomogram.

\section{Ethical approval}

The ethics committee of Sun Yat-sen Memorial Hospital was aware of the present study and agreed that this study did not need ethical approval because it was retrospective with 
deidentified data collected from a database. Patient written informed consent is not usually required for data collection and research purposes if any step involved in collecting patient information or research does not compromise the quality of treatment or confidentiality of the patient.

\section{Results}

Totals of 1,193 and 499 eligible patients were included in the training and validation cohorts, respectively. Clinicopathological data are presented in Table 1. At least one initial positive margin was present in $19.4 \%$ and $25.5 \%$ of the patients in the training and validation cohorts, respectively. Differences in clinicopathological features between cohorts were observed for preoperative size $(P=0.001)$, preoperative lymph-node status $(P=0.007)$, NAC $(P=0.016)$, presence of

Table I Clinicopathological characteristics of training group and validation group

\begin{tabular}{|c|c|c|c|}
\hline \multirow[t]{2}{*}{ Characteristics } & \multirow{2}{*}{$\begin{array}{l}\text { Training } \\
\text { group } \\
(n=l, 193)\end{array}$} & \multirow{2}{*}{$\begin{array}{l}\text { Validation } \\
\text { group } \\
(n=499)\end{array}$} & \multirow[t]{2}{*}{$P$-value } \\
\hline & & & \\
\hline Age, years & $47.9 \pm 10.9$ & $47.4 \pm 10.2$ & \\
\hline \multicolumn{3}{|l|}{ Tumor } & 0.072 \\
\hline Left & 631 (52.9\%) & $240(48.1 \%)$ & \\
\hline Right & $562(47.1 \%)$ & 259 (50.9\%) & \\
\hline \multicolumn{3}{|c|}{ Preoperative size } & 0.001 \\
\hline$\leq 2 \mathrm{~cm}$ & 595 (49.9\%) & $27 \mid(54.3 \%)$ & \\
\hline $2-5 \mathrm{~cm}$ & $432(36.2 \%)$ & 191 (38.3\%) & \\
\hline Unknown & $166(13.9 \%)$ & $37(7.4 \%)$ & \\
\hline \multicolumn{3}{|l|}{ Histology type } & 0.058 \\
\hline Ductal & I,029 (86.3\%) & 409 (82.0\%) & \\
\hline Lobular & 42 (3.5\%) & $27(5.4 \%)$ & \\
\hline Specified & $122(10.2 \%)$ & $63(12.6 \%)$ & \\
\hline \multicolumn{3}{|l|}{ CN stage } & 0.007 \\
\hline Positive & 201 (I6.8\%) & II 12 (22.4\%) & \\
\hline Negative & 992 (83.2\%) & 387 (77.6\%) & \\
\hline \multicolumn{3}{|l|}{ HR } & 0.124 \\
\hline Positive & $\mathrm{I}, 026$ (86.0\%) & $443(88.8 \%)$ & \\
\hline Negative & 167 (14.0\%) & $56(11.2 \%)$ & \\
\hline \multicolumn{3}{|l|}{ HER2 status } & 0.376 \\
\hline Positive & 277 (23.2\%) & $106(21.2 \%)$ & \\
\hline Negative & 916 (78.8\%) & 393 (78.8\%) & \\
\hline \multicolumn{3}{|c|}{ Initial margin status } & 0.008 \\
\hline Positive & $232(19.4 \%)$ & $126(25.2 \%)$ & \\
\hline Negative & $96 \mid(80.6 \%)$ & 373 (74.8\%) & \\
\hline \multicolumn{3}{|c|}{ Suspicion of multifocality } & 0.436 \\
\hline Presence & $79(6.6 \%)$ & $28(5.6 \%)$ & \\
\hline Absence & I, I I 4 (93.4\%) & $47 I(94.4 \%)$ & \\
\hline \multicolumn{3}{|c|}{ Neoadjuvant chemotherapy } & 0.016 \\
\hline Yes & $123(10.3 \%)$ & $72(14.4 \%)$ & \\
\hline No & $1,070(89.7 \%)$ & $427(85.6 \%)$ & \\
\hline \multicolumn{4}{|c|}{ Presence of DCIS } \\
\hline Yes & 231 (19.5\%) & $53(10.6 \%)$ & $<0.001$ \\
\hline No & $962(80.6 \%)$ & $446(89.4 \%)$ & \\
\hline
\end{tabular}

Abbreviations: DCIS, ductal carcinoma in situ; CN, clinical node; HR, hormone receptor; HER2, human epidermal growth receptor 2 .
DCIS in the core needle biopsy $(P<0.001)$, and initial margin status $(P=0.008)$.

The median age of the entire study population was 47.9 (range $22-86)$ years. In the training cohort $(n=1,193)$, 232 of $1,193(19.4 \%)$ patients had positive initial margins. A total of 99 patients were directly converted to mastectomy intraoperatively. Univariate and multivariate analyses (Table 2) suggested that a preoperative tumor size of $2-5 \mathrm{~cm}$ (vs $\leq 2 \mathrm{~cm}$, OR $1.49,95 \%$ CI $1.06-2.12 ; P=0.024)$, preoperative positive lymph-node status (OR 6.56, 95\% CI 4.61-9.31; $P<0.001$ ), suspicion of multifocality (OR 1.84, 95\% CI 1.05-3.23; $P=0.033$ ), positive HR (OR 2.07, 95\% CI 1.25-3.42; $P=0.005$ ), and positive HER2 (OR 1.96, 95\% CI 1.38-2.78; $P<0.001)$ were significantly associated with positive margins. NAC was significant in the univariate analysis (OR 2.3, 95\% CI 1.53-3.45; $P<0.001$ ), but not in the multivariate analysis.

A nomogram was developed using the significant predictors (Figure 1). Area under the curve (AUC) values were 0.72 (95\% CI $0.68-0.76)$ and 0.69 (95\% CI 0.64-0.75) in the training and validation cohorts, respectively (Figure 2). In addition, Youden's index was 0.38 , and the cutoff value that achieved this maximum was 40 points. Calibration plots revealed that predictive probability was consistent with actual probability in both the training cohort (Hosmer-Lemeshow goodness of fit, $\chi^{2}=3.73, P=0.810$ ) and the validation cohort (Hosmer-Lemeshow goodness of fit, $\chi^{2}=13.54, P=0.06$ ) (Figure 3). For patients with NAC, AUC values were 0.81 (95\% CI 0.74-0.90) and 0.75 (95\% CI 0.63-0.87) in the training and validation cohorts, respectively, and predictive probability was consistent with actual probability in the training cohort (Hosmer-Lemeshow goodness of fit, $\chi^{2}=11.17$, $P=0.193$ ) and the validation cohort (Hosmer-Lemeshow goodness of fit, $\chi^{2}=6.07, P=0.639$ ) (data not shown).

\section{Discussion}

After BCS, a second operation for reexcision is common if surgical margins are positive after surgery. In the US, where FSA is not routinely performed, approximately $37.9 \%$ of examined patients receive reexcision after BCS. ${ }^{22}$ The American Society of Breast Surgeons proposed a "toolbox" to decrease the reexcision rate. The toolbox consisted of nine recommendations (eg, compliance with Society of Surgical Oncology-American Society for Radiation Oncology guidelines, use of oncoplastic surgery, and intraoperative specimen imaging). ${ }^{23}$ With the use of these tools, the rate of second surgery was reduced to $21 \%$, according to a recent survey by the American Society of Breast Surgeons. ${ }^{24}$ Among these tools, intraoperative FSA is one of the most popular methods and 
Table 2 Variables in model after univariate and multivariate logistic regression analysis

\begin{tabular}{|c|c|c|c|c|c|c|}
\hline \multirow[t]{2}{*}{ Variables } & \multicolumn{3}{|c|}{ Univariate } & \multicolumn{3}{|c|}{ Multivariate } \\
\hline & OR & $95 \% \mathrm{Cl}$ & $P$-value & OR & $95 \% \mathrm{Cl}$ & $P$-value \\
\hline \multicolumn{7}{|l|}{ Preoperative size } \\
\hline $2-5 \mathrm{vs} \leq 2 \mathrm{~cm}$ & 1.63 & I.19-2.22 & 0.002 & 1.49 & $1.06-2.12$ & 0.024 \\
\hline Unknown vs $\leq 2 \mathrm{~cm}$ & 1.18 & $0.75-1.84$ & 0.470 & 1.49 & $0.93-2.41$ & 0.100 \\
\hline \multicolumn{7}{|l|}{ CN stage } \\
\hline Positive vs negative & 6.64 & $4.76-9.25$ & $<0.001$ & 6.56 & $4.62-9.32$ & $<0.001$ \\
\hline \multicolumn{7}{|l|}{ HR } \\
\hline Positive vs negative & 1.70 & $1.06-2.72$ & 0.029 & 2.07 & $1.25-3.42$ & 0.005 \\
\hline \multicolumn{7}{|l|}{ HER2 status } \\
\hline Positive vs negative & 1.84 & $1.23-2.43$ & $<0.001$ & 1.96 & $1.38-2.78$ & $<0.001$ \\
\hline \multicolumn{7}{|c|}{ Suspicion of multifocality } \\
\hline Presence vs absence & 2.16 & I.32-3.54 & 0.002 & 1.84 & $1.05-3.23$ & 0.033 \\
\hline \multicolumn{7}{|c|}{ Neoadjuvant chemotherapy } \\
\hline Yes vs no & 2.3 & $1.53-3.45$ & $<0.001$ & 1.38 & $0.86-2.21$ & 0.180 \\
\hline \multicolumn{7}{|l|}{ Presence of DCIS } \\
\hline Yes vs no & 1.003 & $0.70-1.44$ & 0.988 & & & \\
\hline
\end{tabular}

Abbreviations: CN, clinical node; DCIS, ductal carcinoma in situ; HR, hormone receptor; HER2, human epidermal growth receptor 2.

has been used in many institutions. A meta-analysis showed that FSA had greater accuracy than ultrasound assessment, specimen radiography, and optical spectroscopy. ${ }^{25}$ However, our studies have revealed a $15 \%-25 \%$ rate of initial margin positivity during intraoperative FSA. ${ }^{16,26,27}$ These patients typically require intraoperative reexcision, with another waiting period for the second FSA of the new margin. Therefore, we developed a nomogram with preoperatively available variables as predictors to estimate the risk of initial margin positivity.

The clinical utility of this nomogram depends on the initial margin positivity rate that is acceptable to both the surgeons and the patients. In our institution, we routinely perform FSA for all BCS patients. The current second-surgery rate due to false-negative FSA results is approximately $3.5 \%$ in our institution. To reduce waiting time and medical costs, we are

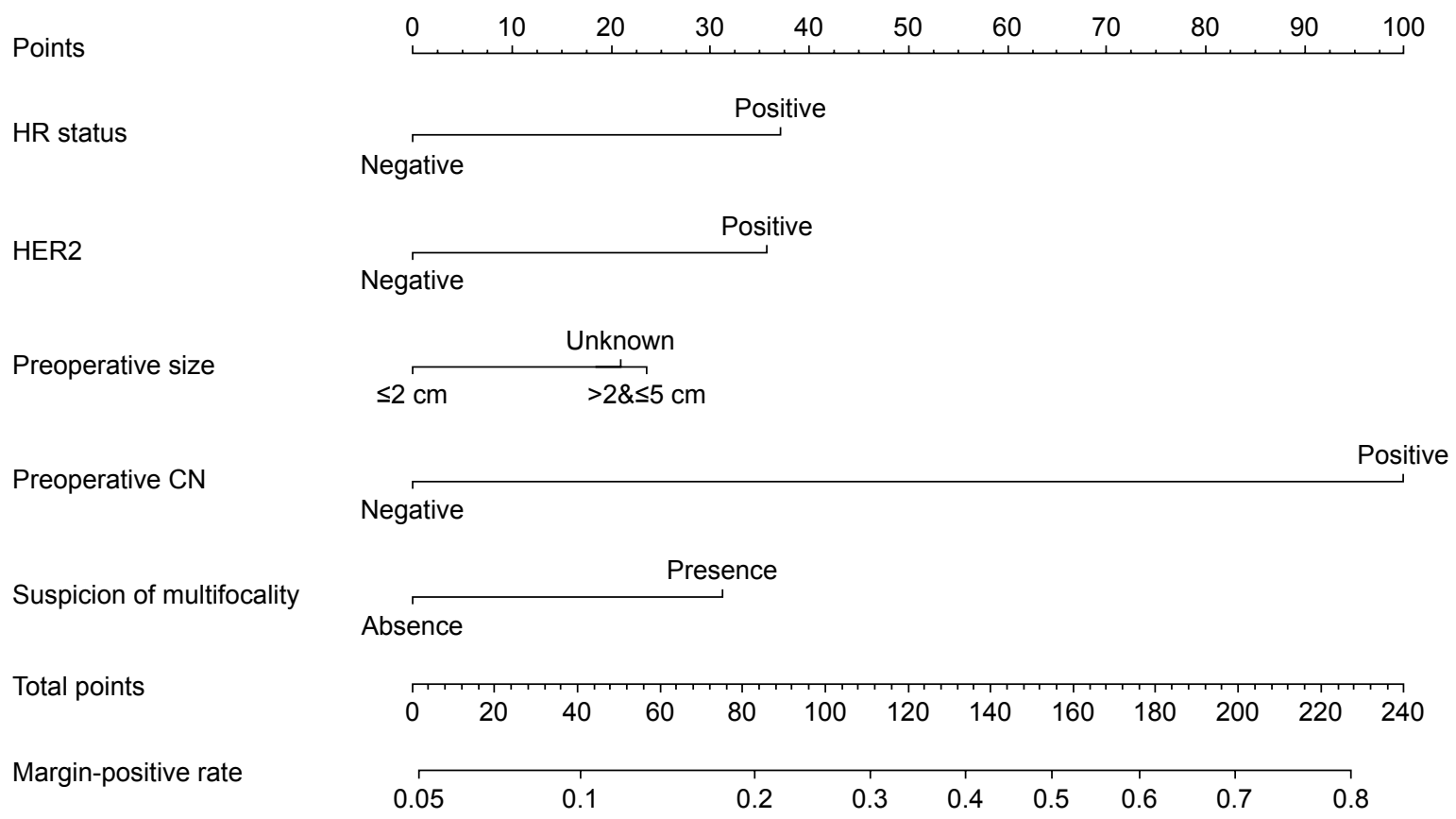

Figure I Nomography to predict probability of positive initial margins.

Notes: For each patient, we calculated points for corresponding variables and summed them. The predicted margin-positive rate can be estimated based on the total points for each patient.

Abbreviations: CN, clinical node; HR, hormone receptor; HER2, human epidermal growth receptor 2 . 


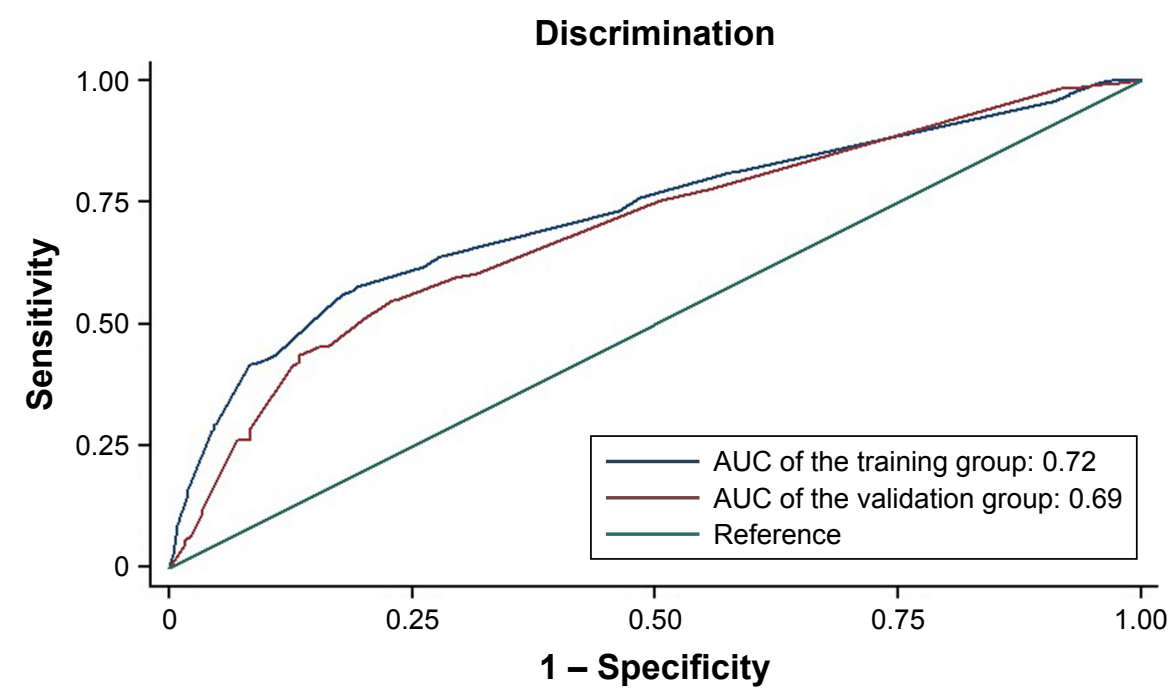

Figure 2 Nomography for training and validation groups. The AUC indicated the discriminative power of the nomogram. Abbreviation: AUC, area under the curve (receiver-operating characteristic).

considering foregoing FSA for intraoperative margin assessment in selected patients. From our perspective, $<10 \%$ risk of second surgery is acceptable, because the second-surgery rate is approximately $30 \%-40 \%$ in the US, where FSA is not routinely performed. Therefore, with our nomogram, we can preoperatively identify approximately $38 \%$ of patients with $<10 \%$ predicted risk of having initial positive margins, and foregoing FSA for margin assessment is reasonable in these patients. We can also identify approximately $16 \%$ of patients with $>30 \%$ risk of having initial margin positivity.
For these patients, we can recommend oncoplastic breast surgery with wider margins. Because the accuracy of the FSA is high (false-positive rate 0 and false-negative rate $3.5 \%$ ) in our institution, this nomogram may also be useful for institutions where FSA is not routinely performed. Wider surgical margins can be considered for patients with a high risk of having positive margins.

Several models have been developed by other studies ${ }^{12,28-30}$ to predict the probability of positive margins (Table 3). Our nomogram differs from previous models. First, our study
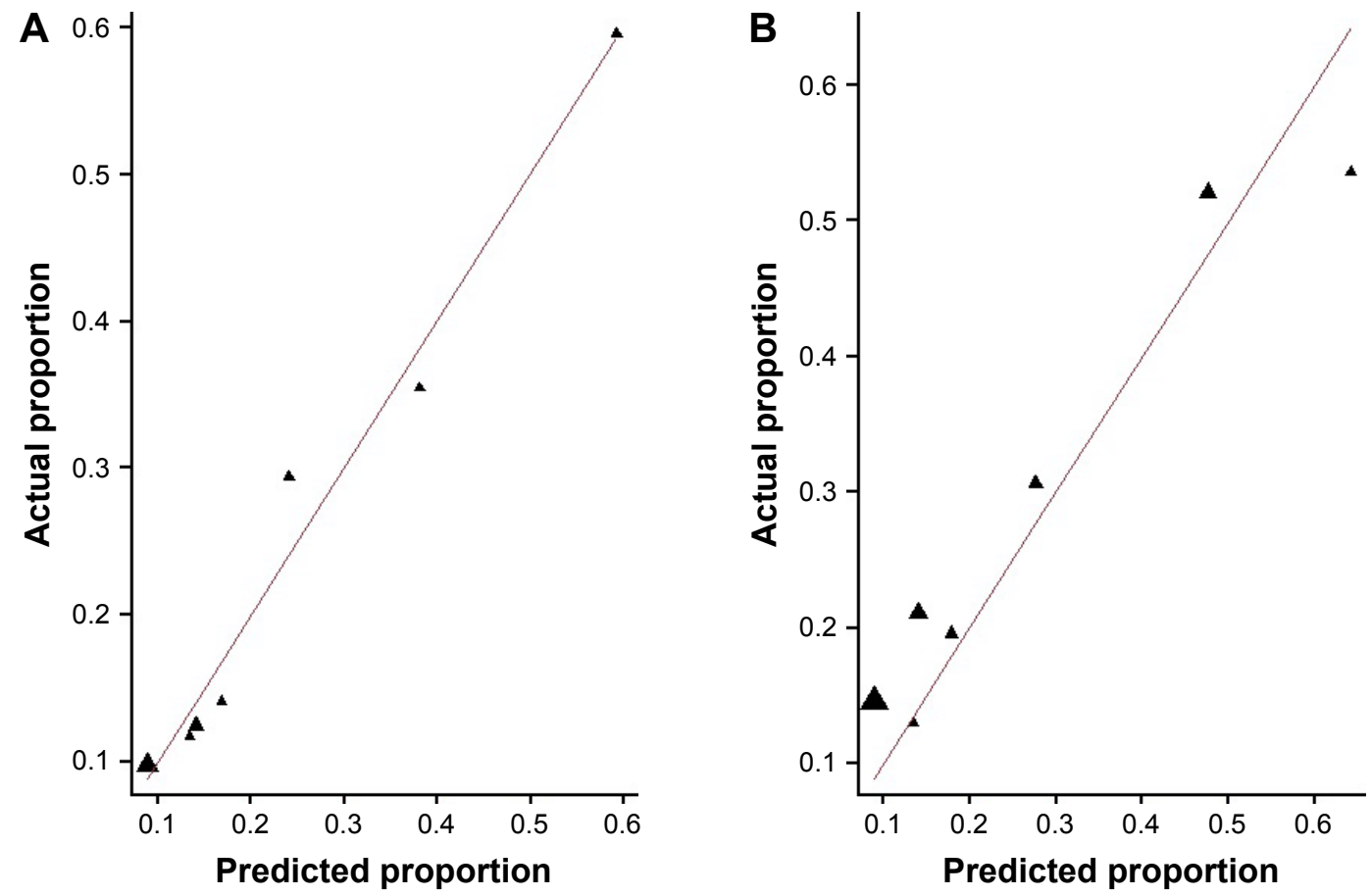

Figure 3 Calibration of nomography in training group $(\mathbf{A})$ and validation group (B). Mean predicted probabilities plotted against actual probabilities of positive margins. 
Table 3 Comparison with previous model predicting likelihood of positive margin

\begin{tabular}{|c|c|c|c|c|c|c|c|c|c|}
\hline \multirow[t]{2}{*}{ Study } & \multirow[t]{2}{*}{ Population } & \multirow[t]{2}{*}{ Assessment } & \multirow[t]{2}{*}{ NAC } & \multirow{2}{*}{$\begin{array}{l}\text { Rate of } \\
\text { positive } \\
\text { margin }\end{array}$} & \multirow[t]{2}{*}{ Variables in model } & \multirow[t]{2}{*}{ OR } & \multirow[t]{2}{*}{$95 \% \mathrm{Cl}$} & \multicolumn{2}{|l|}{ AUC } \\
\hline & & & & & & & & Model & Validation \\
\hline \multirow[t]{8}{*}{$\begin{array}{l}\text { Shin } \\
\text { et } \mathrm{al}^{30}\end{array}$} & $\begin{array}{l}\text { Patients with invasive } \\
\text { or in situ palpable }\end{array}$ & Lump margin & Excluded & $\begin{array}{l}15 \mid \text { of } I, 034 \\
(14.6 \%)\end{array}$ & $\begin{array}{l}\text { Microcalcification on mammography } \\
\text { Breast density on mammography }\end{array}$ & 1.57 & $1.04-2.39$ & 0.823 & 0.846 \\
\hline & and impalpable & & & & Type 2 & 1.59 & $0.53-4.81$ & & \\
\hline & breast cancer & & & & Type 3 & 1.61 & $0.56-4.62$ & & \\
\hline & undergoing BCS & & & & Type 4 & 4.52 & $1.57-12.95$ & & \\
\hline & & & & & $>0.5 \mathrm{~cm}$ difference on & 10.0 & $6.50-15.39$ & & \\
\hline & & & & & MRI ultrasound & & & & \\
\hline & & & & & DCIS present on needle biopsy & 1.58 & $\mathrm{I} .0 \mathrm{I}-2.45$ & & \\
\hline & & & & & $\begin{array}{l}\text { Lobular component on } \\
\text { needle biopsy }\end{array}$ & 3.99 & $1.31-12.12$ & & \\
\hline \multirow{11}{*}{$\begin{array}{l}\text { Pleijhuis } \\
\text { et al }{ }^{12,28}\end{array}$} & Patients with & Lump margin & Excluded & 233 of $I, 185$ & Suspicion of multifocal disease & 2.81 & $1.30-6.06$ & 0.70 & 0.69 \\
\hline & TI-T2 palpable and & & & $(19.7 \%)$ & Preoperative MRI scan absent & 1.80 & $1.02-3.18$ & & \\
\hline & impalpable breast & & & & Positive preoperative $\mathrm{N}$ stage & 1.73 & $0.97-3.07$ & & \\
\hline & cancer undergoing & & & & Impalpable tumor & 1.51 & $1.07-2.13$ & & \\
\hline & BCS & & & & Microcalcifications on mammogram & 1.37 & $0.95-2.00$ & & \\
\hline & & & & & Preoperative T2 stage & 1.33 & $0.87-2.02$ & & \\
\hline & & & & & Presence of DCIS component & 3.11 & $2.19-4.42$ & & \\
\hline & & & & & Lobular histology & 2.90 & $|.7|-4.9 \mid$ & & \\
\hline & & & & & ER-positive & 1.80 & $1.04-3.13$ & & \\
\hline & & & & & Elston grade 3 & 1.44 & $0.96-2.16$ & & \\
\hline & & & & & Breast density on mammogram & 1.22 & $1.00-1.49$ & & \\
\hline \multirow{6}{*}{$\begin{array}{l}\text { Barentsz } \\
\text { et } \mathrm{al}^{29}\end{array}$} & Patients with & Lump margin & Excluded & 69 of 576 & Microcalcifications on mammogram & 2.14 & $1.22-3.77$ & 0.70 & 0.69 \\
\hline & impalpable breast & & & $(12.0 \%)$ & Invasive tumor size & 1.75 & $1.20-2.56$ & & \\
\hline & cancer undergoing & & & & Presence of DCIS component & 2.61 & $1.41-4.82$ & & \\
\hline & $\mathrm{BCS}$ & & & & on biopsy & & & & \\
\hline & & & & & Bloom-Richardson grade $2 / 3$ & 1.82 & $1.05-3.14$ & & \\
\hline & & & & & Caudal location within breast & 2.40 & $1.35-4.27$ & & \\
\hline \multirow{7}{*}{$\begin{array}{l}\text { Present } \\
\text { study }\end{array}$} & Patients with invasive & Cavity margin & Included & 232 of $I, 193$ & Preoperative tumor size & & & 0.72 & 0.69 \\
\hline & or in situ palpable & & & $(19.4 \%)$ & $2-5 \mathrm{~cm}$ & 1.57 & $1.12-2.20$ & & \\
\hline & and non-palpable & & & & Unknown & 1.50 & $0.93-2.42$ & & \\
\hline & breast cancer & & & & Positive preoperative $\mathrm{N}$ stage & 6.83 & $4.83-9.66$ & & \\
\hline & undergoing BCS & & & & HR-positive & 2.04 & $1.23-3.37$ & & \\
\hline & & & & & Positive HER2 & 1.99 & $|.4|-2.82$ & & \\
\hline & & & & & Suspicion of multifocality & 1.83 & $1.04-3.21$ & & \\
\hline
\end{tabular}

Abbreviations: AUC, area under the curve; BCS, breast-conserving surgery; CN, clinical node; DCIS, ductal carcinoma in situ; HR, hormone receptor; HER2, human epidermal growth receptor 2; NAC, neoadjuvant chemotherapy.

population included patients with NAC, who were excluded in previous studies. ${ }^{28-30} \mathrm{NAC}$ has the potential to shrink the tumor and increase the possibility of breast conservation. ${ }^{31,32}$ Because NAC may shrink the tumor in a mosaic/honeycomb pattern, ${ }^{33}$ margin assessment may be challenging in these patients. Our study, together with our previous report, demonstrated that patients with NAC exhibited a significantly increased margin-positivity rate. ${ }^{27}$ In our study, 123 (10.3\%) and $72(14.4 \%)$ patients received NAC in the training and validation cohorts, respectively. The subgroup analysis revealed that the new nomogram was also effective for patients with NAC. Second, during BCS, we assessed only the cavity margin and not the lumpectomy (resection) margins reported by National Surgical Adjuvant Breast and Bowel Project B06. ${ }^{34}$ Our study and others revealed potential discrepancies between the cavity- and lumpectomy-margin statuses. ${ }^{27,35}$ Given that cavity-margin status is more prognostic than lumpectomy-margin status, ${ }^{27,35}$ a model must be developed to predict the former.

Third, previous models included the presence of DCIS as an important predictor. ${ }^{28-30}$ However, the presence of DCIS determined by core needle biopsy may not be reliable. Studies have demonstrated that the false-negative rate of DCIS detection by core needle biopsy (defined as absence of DCIS in the core needle biopsy and presence of DCIS in the final pathological examination) ranges between $36 \%$ and $54 \%{ }^{36,37}$ Pleijhuis et al used DCIS status from the final pathological examination as a predictor, rather than the biopsy pathological examination. ${ }^{28}$ In a study to validate their model, Alves-Ribeiro et al reported that only 166 (68.9\%) 
of the 241 patients classified by core needle biopsy had a congruent preoperative evaluation of the presence of DCIS. ${ }^{38}$ Additionally, preoperative predictors were more valuable than postoperative variables in predicting initial margin status. These findings implied that this variable was not relatively reliable when used in the predicted model. In our study, we did not identify an association between the presence of DCIS in the core needle biopsy and positive margins. Therefore, this variable was not included as a predictor. Fourth, Shin et al developed a model with a relatively high AUC $(0.823,95 \% \text { CI 0.785-0.862) })^{30}$ However, whether their nomogram can be externally validated in other populations is unknown. In addition, the difference in size based on magnetic resonance imaging (MRI) and ultrasound is included as a predictor. However, MRI is not a routine examination for patients without NAC according to the National Comprehensive Cancer Network guidelines, which reduces the applicability of their model. In our study population, few patients received preoperative MRI.

Several limitations of our study should be noted. First, this was a retrospective study with inevitable bias, including the number of FSA samples and the choice of surgery by the surgeons. Second, the study population was collected from a single institution (Sun Yat-sen Memorial Hospital). Additionally, the validation was arguably only internal and not external. However, the training and validation cohorts were actually selected from two independent and different surgical teams located in different branches of the hospital. Their surgical treatment algorithms were different, but were supported by the same pathology department. Therefore, we suggest that the validation group is valid in this study. Third, mammographic breast density may add more value to prediction accuracy, but we did not have this information. Fourth, since cavity- and lumpectomy-margin statuses may not always be concordant, whether the new model can be used to predict lumpectomy-margin status is unclear. Fifth, the margin-positivity rate according to the postoperative pathological examination may be of particular clinical interest. However, because the accuracy rate of FSA is as high as $97 \%$ in our institution, using the initial margin status as the primary end point was also acceptable in this study. A prospective study is needed to confirm our model. Sixth, tumor grade and Ki67 status have been proposed as potential predictive biomarkers for margin status. Since the purpose of the nomogram is to predict margin status, only tumor grading obtained from the core needle biopsy can be used. However, in our institution, the pathologist rarely reports tumor grades based on the specimen from the core needle biopsy. The underlying reason is that the mitotic count is often underestimated, due to the limited specimen volume. Studies have also shown that the histological grade assessed from the core needle biopsy does not concur well with the grade from the surgical excision specimen $(\kappa=0.35-0.65) .{ }^{39,40}$ Therefore, we cannot include tumor grade as a predictor. In addition, Ki67 is a well-known prognostic factor. We tried but failed to find any association between Ki67 status and margin status in the univariate analysis. Therefore, Ki67 was not included in the nomogram. Lack of tissue volume from the core needle biopsy may be one factor that limits the interpretation of Ki67 status. Additionally, enormous variation in analytical practices markedly limits the value of Ki67. ${ }^{41}$ Therefore, more studies are needed to investigate the role of Ki67 in predicting margin status. Furthermore, how this nomogram is applicable to other institutions in different regions or countries is unclear, and a multicentric study is needed to assess its external validity.

\section{Conclusion}

We developed a nomogram based on preoperatively available information to predict the risk of having positive initial margins during BCS. This nomogram will be useful in clinical decision-making.

\section{Acknowledgments}

This study was supported by the National Natural Science Foundation of China (grants 81402201 and 81372817), National Natural Science Foundation of Guangdong Province (grant 2014A030310070), and grant 163 (2013) from the Key Laboratory of Malignant Tumor Molecular Mechanism and Translational Medicine of the Guangzhou Bureau of Science and Information Technology.

\section{Disclosure}

The authors report no conflicts of interest in this work.

\section{References}

1. Fisher ER, Anderson S, Redmond C, Fisher B. Ipsilateral breast tumor recurrence and survival following lumpectomy and irradiation: pathological findings from NSABP protocol B-06. Semin Surg Oncol. 1992;8(3):161-166.

2. Fisher B, Anderson S, Bryant J, et al. Twenty-year follow-up of a randomized trial comparing total mastectomy, lumpectomy, and lumpectomy plus irradiation for the treatment of invasive breast cancer. $N$ Engl $J$ Med. 2002;347(16):1233-1241.

3. Veronesi U, Cascinelli N, Mariani L, et al. Twenty-year follow-up of a randomized study comparing breast-conserving surgery with radical mastectomy for early breast cancer. $N$ Engl J Med. 2002;347(16):1227-1232.

4. Vila J, Gandini S, Gentilini O. Overall survival according to type of surgery in young ( $\leq 40$ years) early breast cancer patients: a systematic meta-analysis comparing breast-conserving surgery versus mastectomy. Breast. 2015;24(3):175-181. 
5. Gentilini O, Botteri E, Rotmensz N, et al. Breast-conserving surgery in 201 very young patients (<35 years). Breast. 2010;19(1):55-58.

6. Lowery AJ, Kell MR, Glynn RW, Kerin MJ, Sweeney KJ. Locoregional recurrence after breast cancer surgery: a systematic review by receptor phenotype. Breast Cancer Res Treat. 2012;133(3):831-841.

7. Nguyen PL, Taghian AG, Katz MS, et al. Breast cancer subtype approximated by estrogen receptor, progesterone receptor, and HER-2 is associated with local and distant recurrence after breast-conserving therapy. J Clin Oncol. 2008;26(14):2373-2378.

8. Gangi A, Chung A, Mirocha J, Liou DZ, Leong T, Giuliano AE. Breast-conserving therapy for triple-negative breast cancer. JAMA Surg. 2014;149(3):252-258.

9. Moran MS, Schnitt SJ, Giuliano AE, et al. Society of Surgical Oncology-American Society for Radiation Oncology consensus guideline on margins for breast-conserving surgery with whole-breast irradiation in stages I and II invasive breast cancer. J Clin Oncol. 2014; 32(14):1507-1515.

10. Pilewskie M, Ho A, Orell E, et al. Effect of margin width on local recurrence in triple-negative breast cancer patients treated with breastconserving therapy. Ann Surg Oncol. 2014;21(4):1209-1214.

11. Bartelink H, Maingon P, Poortmans P, et al. Whole-breast irradiation with or without a boost for patients treated with breast-conserving surgery for early breast cancer: 20-year follow-up of a randomised phase 3 trial. Lancet Oncol. 2015;16(1):47-56.

12. Pleijhuis RG, Graafland M, de Vries J, Bart J, de Jong JS, van Dam GM. Obtaining adequate surgical margins in breast-conserving therapy for patients with early-stage breast cancer: current modalities and future directions. Ann Surg Oncol. 2009;16(10):2717-2730.

13. Fleming FJ, Hill AD, Mc Dermott EW, O’Doherty A, O'Higgins NJ, Quinn CM. Intraoperative margin assessment and re-excision rate in breast conserving surgery. Eur J Surg Oncol. 2004;30(3):233-237.

14. Bolger JC, Solon JG, Khan SA, Hill AD, Power CP. A comparison of intra-operative margin management techniques in breast-conserving surgery: a standardised approach reduces the likelihood of residual disease without increasing operative time. Breast Cancer. 2015;22(3): 262-268.

15. Jorns JM, Visscher D, Sabel M, et al. Intraoperative frozen section analysis of margins in breast conserving surgery significantly decreases reoperative rates: one-year experience at an ambulatory surgical center. Am J Clinical Pathol. 2012;138(5):657-669.

16. Chen $\mathrm{K}$, Zeng Y, Jia H, et al. Clinical outcomes of breast-conserving surgery in patients using a modified method for cavity margin assessment. Ann Surg Oncol. 2012;19(11):3386-3394.

17. Kurniawan ED, Wong MH, Windle I, et al. Predictors of surgical margin status in breast-conserving surgery within a breast screening program. Ann Surg Oncol. 2008;15(9):2542-2549.

18. Lee ES, Han W, Shin HC, et al. Clinical benefit of nomogram for predicting positive resection margins in breast conserving surgery. Eur $J$ Surg Oncol. 2016;42(8):1169-1175.

19. Bossuyt PM, Reitsma JB, Bruns DE, et al. STARD 2015: an updated list of essential items for reporting diagnostic accuracy studies. $B M J$. 2015;351:h5527.

20. Chen K, Jia W, Li S, et al. Cavity margin status is an independent risk factor for local-regional recurrence in breast cancer patients treated with neoadjuvant chemotherapy before breast-conserving surgery. Am Surg. 2011;77(12):1700-1706.

21. Jacobs TW, Gown AM, Yaziji H, Barnes MJ, Schnitt SJ. Specificity of HercepTest in determining HER-2/neu status of breast cancers using the United States Food and Drug Administration-approved scoring system. J Clin Oncol. 1999;17(7):1983-1987.

22. Morrow M, Jagsi R, Alderman AK, et al. Surgeon recommendations and receipt of mastectomy for treatment of breast cancer. JAMA. 2009; 302(14):1551-1556.

23. Landercasper J, Attai D, Atisha D, et al. Toolbox to reduce lumpectomy reoperations and improve cosmetic outcome in breast cancer patients: the American Society of Breast Surgeons Consensus Conference. Ann Surg Oncol. 2015;22(10):3174-3183.
24. Leff DR, St John ER, Takats Z. Reducing the margins of error during breast-conserving surgery: disruptive technologies or traditional disruptions? JAMA Surg. 2017;152(6):517-518

25. St John ER, Al-Khudairi R, Ashrafian H, et al. Diagnostic accuracy of intraoperative techniques for margin assessment in breast cancer surgery: a meta-analysis. Ann Surg. 2017;265(2):300-310.

26. Jia WJ, Jia HX, Feng HY, Yang YP, Chen K, Su FX. HER2-enriched tumors have the highest risk of local recurrence in Chinese patients treated with breast conservation therapy. Asian Pac J Cancer Prev. 2014;15(1):315-320.

27. Yang $\mathrm{H}$, Jia $\mathrm{W}$, Chen $\mathrm{K}$, et al. Cavity margins and lumpectomy margins for pathological assessment: which is superior in breast-conserving surgery? J Surg Res. 2012;178(2):751-757.

28. Pleijhuis RG, Kwast AB, Jansen L, et al. A validated web-based nomogram for predicting positive surgical margins following breastconserving surgery as a preoperative tool for clinical decision-making. Breast. 2013;22(5):773-779.

29. Barentsz MW, Postma EL, van Dalen T, et al. Prediction of positive resection margins in patients with non-palpable breast cancer. Eur $J$ Surg Oncol. 2015;41(1):106-112.

30. Shin HC, Han W, Moon HG, et al. Nomogram for predicting positive resection margins after breast-conserving surgery. Breast Cancer Res Treat. 2012;134(3):1115-1123.

31. Buchholz TA, Mittendorf EA, Hunt KK. Surgical considerations after neoadjuvant chemotherapy: breast conservation therapy. J Natl Cancer Inst Monogr. 2015;2015(51):11-14.

32. Mamounas EP. Impact of neoadjuvant chemotherapy on locoregional surgical treatment of breast cancer. Ann Surg Oncol. 2015;22(5): $1425-1433$.

33. Akashi-Tanaka S, Fukutomi T, Sato N, et al. The use of contrastenhanced computed tomography before neoadjuvant chemotherapy to identify patients likely to be treated safely with breast-conserving surgery. Ann Surg. 2004;239(2):238-243.

34. Fisher ER, Sass R, Fisher B, Gregorio R, Brown R, Wickerham L. Pathologic findings from the National Surgical Adjuvant Breast Project (protocol 6) - II: relation of local breast recurrence to multicentricity. Cancer. 1986;57(9):1717-1724.

35. Tengher-Barna I, Hequet D, Reboul-Marty J, et al. Prevalence and predictive factors for the detection of carcinoma in cavity margin performed at the time of breast lumpectomy. Mod Pathol. 2009;22(2):299-305.

36. Jimenez RE, Bongers S, Bouwman D, Segel M, Visscher DW. Clinicopathologic significance of ductal carcinoma in situ in breast core needle biopsies with invasive cancer. Am J Surg Pathol. 2000;24(1): $123-128$.

37. Dillon MF, Maguire AA, McDermott EW, et al. Needle core biopsy characteristics identify patients at risk of compromised margins in breast conservation surgery. Mod Pathol. 2008;21(1):39-45.

38. Alves-Ribeiro L, Osorio F, Amendoeira I, Fougo JL. Positive margins prediction in breast cancer conservative surgery: assessment of a preoperative web-based nomogram. Breast. 2016;28:167-173.

39. Knuttel FM, Menezes GL, van Diest PJ, Witkamp AJ, van den Bosch MA, Verkooijen HM. Meta-analysis of the concordance of histological grade of breast cancer between core needle biopsy and surgical excision specimen. Br J Surg. 2016;103(6):644-655.

40. Waaijer L, Willems SM, Verkooijen HM, et al. Impact of preoperative evaluation of tumour grade by core needle biopsy on clinical risk assessment and patient selection for adjuvant systemic treatment in breast cancer. Br J Surg. 2015;102(9):1048-1055.

41. Dowsett M, Nielsen TO, A'Hern R, et al. Assessment of Ki67 in breast cancer: recommendations from the International Ki67 in Breast Cancer working group. J Natl Cancer Inst. 2011;103(22):1656-1664. 
OncoTargets and Therapy

\section{Publish your work in this journal}

OncoTargets and Therapy is an international, peer-reviewed, open access journal focusing on the pathological basis of all cancers, potential targets for therapy and treatment protocols employed to improve the management of cancer patients. The journal also focuses on the impact of management programs and new therapeutic agents and protocols on

patient perspectives such as quality of life, adherence and satisfaction The manuscript management system is completely online and includes a very quick and fair peer-review system, which is all easy to use. Visit http://www.dovepress.com/testimonials.php to read real quotes from published authors.

Submit your manuscript here: http://www.dovepress.com/oncotargets-and-therapy-journal 